\title{
To the rationalization of the constructive solutions of the bridge supports
}

\author{
Olena Petrova $^{1 *}$, Olena Lugchenko ${ }^{1}$, Mohammad Hammud ${ }^{1}$, and Ali Nazhem ${ }^{1}$ \\ ${ }^{1}$ Department of Building Structures, O.M. Beketov National University of Urban Economy in \\ Kharkiv, 61002, Kharkiv, Ukraine
}

\begin{abstract}
Reduction of material consumption and labor intensity of building structures is a topical task for both theory and practice of building production. Today there are many ways to achieve proper economic effect while reconstruction or erection of building and structures. One of them is to rationalize well-known constructive solutions using as criteria the value of density of potential energy of deformation of structures. In this connection, the new constructive solution for short massive supports with outer reinforcement made of expanded metal sheet and frames (reinforcing rings) which are used in construction of road and railway bridges and highrise buildings is proposed in this paper. The theoretical approach for the definition of its rational parameters is given. Numerical analysis of the highlighted method is also carried out. As a result of study the increase in the bearing capacity in concrete pillars made in rational mesh clips with and without frames was obtained.
\end{abstract}

\section{Introduction}

During the last decades, the reduction of the material consumption of building structures, while maintaining their high rigidity and strength characteristics has been the major task for both theoretical research and experimental verification. At present, this objective can be achieved either by introducing absolutely new effective constructive solutions, or by improving and rationalizing the already known ones. One of the promising ways of this direction, in particular, is the use of a high-tech expanded metal sheet as an external reinforcement of structures.

The use of external sheet reinforcement in the form of a mesh outer ring can simultaneously perform protective, technological and power functions. For example, if we consider steel bridge supports or heavily loaded supporting columns of high-rise buildings and structures, the use of such a clip allows us to limit the displacement of concrete inside it in the transverse direction. In turn, the three-axially compressed concrete located inside the clip increases the bearing capacity of the support itself. This fact is especially important for supports experiencing a high level of both static and dynamic loading. In addition, the use of expanded metal sheet allows achieving of about 30-60\% reduction of metal consumption, which, in turn, leads to a reduction of the weight of the whole structure.

\footnotetext{
* Corresponding author: petrova.bcd@gmail.com
} 


\section{Formulation and solution of the problem}

Currently, the works by V.Shmukler and O.Sinkovskaya [1] are known, in which short reinforced concrete supports with external reinforcement made of expanded metal sheet are considered. The effectiveness of the constructive solution proposed by the authors is confirmed experimentally. Based on the established rational tilt angle of the generatrix of the cell of expanded metal sheet, in this work we propose a new design solution of the outer ring with reinforcing frames as well as a procedure for selecting of rational parameters for such an element.

In this connection, the structure of a circular cylindrical short quasi-centrally loaded support (Fig. 1), which is made of concrete encased in a steel mesh clip, is considered. This clip is manufactured by non-waste technology from expanded metal sheet. The connection of the edges of the clip is carried out by welding. To increase the crimping effect of the clip special rings, including reinforcing steel called "the frames", are used. They are installed with a constant step $l$ and have a cross-sectional area $A_{f}$. Since the column is considered to be loaded with a quasi-central load, the problem is assumed to be axial symmetric one.

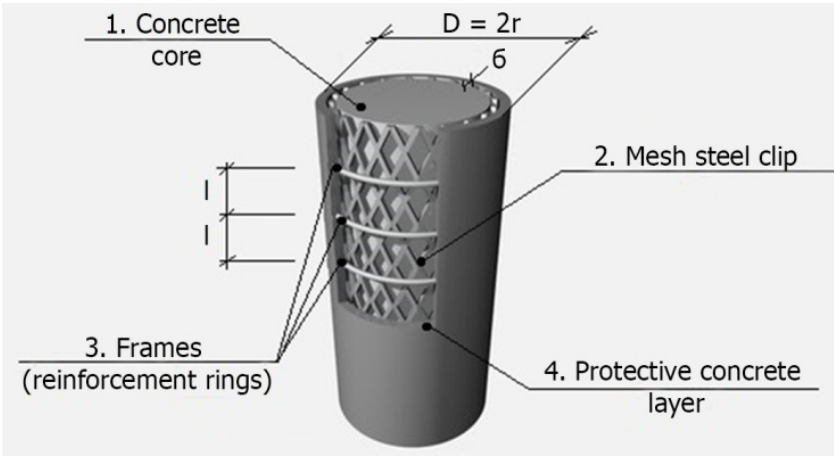

Fig. 1. Cylindrical support with a mesh clip and reinforcing frames.

The geometric dimensions of the column (first of all, the $H / D$ ratio, where $H$ is the height of the column, and $D$ is its diameter) are chosen in such a way that the possibility of loss of stability of equilibrium is excluded. When considering the design model of such support, it is considered that longitudinal compressive forces are applied to the ends of the concrete core (Fig. 2).
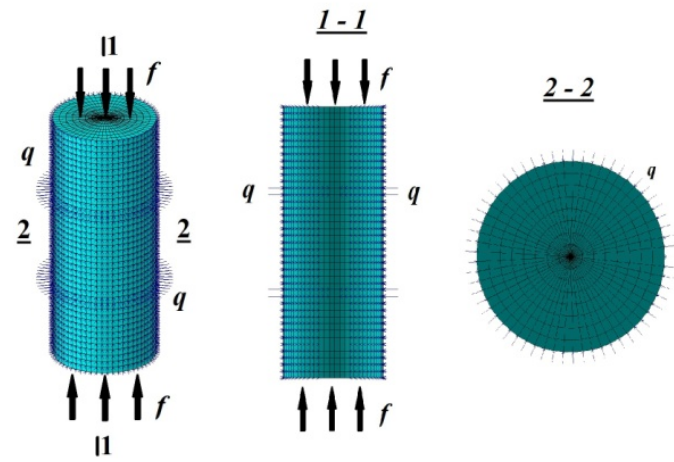

Fig. 2. 3D finite-element calculation model of the support.

As is known, the efficiency of reinforcing of the concrete pillar via frame sharply decreases in the case of appearance of the accidental (calculated) eccentricities. However, it 
is somewhat mitigating that the design we propose here is intended to be used for erecting pillars of road and railway bridges, as well as columns of high-rise buildings, which are usually projected in a form of inflexible, loaded, mainly with longitudinal forces applied with low eccentricity supports.

While solving the problem of searching for a rational constructive solution of the proposed support, the following simplifying hypotheses and assumptions are accepted:

- massive short rods (with flexibility $\lambda \leq 10$ ) are considered;

- material properties are specified by the "tension - deformation" indicator diagrams;

- materials are considered conditionally nonlinearly elastic;

- the volume deformation is approximately taken to be equal to $\varepsilon_{0}=0$. In this case, the third hypothesis of the theory of small elastic plastic deformations about the coincidence of the indicator diagram of the material with the dependence of "tension intensity - deformation intensity" is valid;

- the stress-strain state of the element is considered quasi-homogeneous; that means the assumption of $e=$ const, where $e$ is the density of the potential energy of the deformations (DPED);

- the compressive stresses $f$ are assumed to be uniformly distributed along the ends of the element and are applied only to its concrete part;

- it is considered (for the reserve) that only the radial stresses occur at the point of contact of the concrete core and metal clip;

- the value of the normalized potential energy of deformations (NPED) is adopted as the point of conditional resilience for a concrete element;

- a linear dependence between PED and material consumption is assumed.

Further, it is necessary to identify the criterion of the problem and introduce some constraints.

As a criterion of the problem, the condition for maximizing the value of the contact pressure between the frame and the metal clip is adopted here:

$$
P \rightarrow \text { sup }
$$

where $P$ is the circumferential contact pressure between the frame and the mesh.

Restrictions are accepted as following:

- $\quad$ boundary conditions - the ends of the element are free from fastenings [2];

- $\quad$ isoperimetric condition $e=$ const;

- $\quad$ condition of compatibility of deformation on the whole range of loading

$$
\varepsilon_{c}=\varepsilon_{s},
$$

where $\varepsilon_{c}, \varepsilon_{s}$ is the deformation of the concrete and the meshclip in their contact zone.

Further, it is conventionally considered (because of the reduced thickness of the mesh in comparison with the radius of the concrete core) that the pressure $P$ is completely transferred to the concrete.

In accordance with the accepted hypothesis, the radius of the element is chosen from the condition:

$$
\lambda=\frac{4 H}{D} \leq 10
$$

Basing on specified height of the element and the radius determined from (4), the vector of the variable (controlling) parameters will have the form:

$$
\{\overline{\mathrm{x}}\}^{\mathrm{T}}=\left\{h_{\text {red }} ; l ; d\right\}
$$

where $h_{\text {red }}$ is the reduced thickness of the mesh clip, $l$ is the step of the reinforcing rings (frames) and dis the diameter of the cross-section of the frames.

Let the reduced thickness be 


$$
h_{\text {red }}=\alpha \cdot r \text {, }
$$

where $r$ is the radius of the concrete core, $D=2 r ; \alpha \in\left(\alpha_{\min } ; \alpha_{\max }\right)$ is the coefficient, and $\alpha_{\min }, \alpha_{\max }$ are known constants, and

$$
A_{f}=\frac{\pi d^{2}}{4}, \text { where }
$$

$A_{f}$ is a cross-sectional area of the frame ring, $d \in\left(d_{\min } ; d_{\max }\right), d_{\min }, d_{\max }$ are known constants, and, finally,

$$
l=\gamma \cdot r
$$

$\gamma \in\left(\gamma_{\min } ; \gamma_{\max }\right)$ is the coefficient, $\gamma_{\min }, \gamma_{\max }$ are known constants.

Considering (6) and (7) we can set:

$$
\{\overline{\mathrm{x}}\}^{\mathrm{T}}=\{\alpha ; \gamma ; d\}
$$

under the next constraints

$$
\begin{array}{r}
\alpha_{\min } \leq \alpha \leq \alpha_{\max } \\
\gamma_{\min } \leq \gamma \leq \gamma_{\max } \\
d_{\min } \leq d \leq d_{\max }
\end{array}
$$

To achieve stated goal, it is also necessary to solve a number of subsidiary tasks.

Thus, using the solution of the Lame problem, we can write

$$
q=b \cdot f,
$$

where $q$ is acontact radial stresses between the concrete core and the mesh clip;

$$
b=\frac{\mu_{c} n \omega}{\left(1-\mu_{c}\right) n \omega+\mu_{s}},
$$

where $\mu_{c}, \mu_{s}$ are the Poisson coefficients of concrete and steel, respectively;

$$
n=\frac{E_{s}}{E_{c}}, \omega=\frac{h_{r e d}}{r}=\frac{\alpha r}{r}=\alpha
$$

where $E_{c}, E_{s}$ are the secant modulus of deformation of the first kind of concrete and steel.

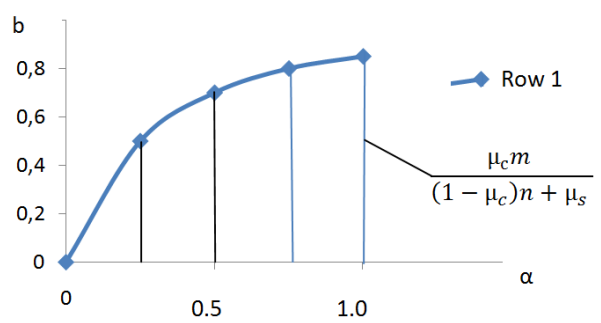

Fig. 3. Dependence $b_{(\alpha)}$.

Having the expression for the contact distributed stresses (10) and using the solution of S.P. Tymoshenko, who determines the radial contact annular stresses that arise between the frames, located with the same constant step, and mesh clip, after the transformations, we obtain [3]:

$$
P=b \cdot \psi \cdot f
$$




$$
\begin{gathered}
\psi=\frac{2}{\beta} \cdot \frac{1}{\frac{2 h_{r e d}+\frac{\operatorname{sh} \beta l+\sin \beta l}{\beta A_{f}}+\frac{\operatorname{ch} \beta l-\cos \beta l}{}}{\beta}}, \\
\beta=4 \sqrt{\frac{3\left(1-\mu_{s}\right)}{r^{2} \cdot h_{\text {red }}^{2}}}
\end{gathered}
$$

Taking into account designations accepted previously, and the fact that $\mu_{s}=0,3$

$$
\beta=\frac{1,2}{r \sqrt{\alpha}}
$$

The expression for $\psi(13)$ will take the form:

$$
\psi=1,7 \cdot r \sqrt{\alpha} \cdot \frac{1}{2.12 \alpha \sqrt{\alpha} \cdot \frac{r^{2}}{d^{2}}+\frac{\operatorname{sh} \bar{\beta}+\sin \bar{\beta}}{\operatorname{ch} \bar{\beta}-\cos \bar{\beta}}},
$$

where $\bar{\beta}=\beta l=\frac{1,2 \gamma}{l \sqrt{\alpha}}$.

Let's set the following designations:

$$
\begin{aligned}
& \mathrm{c}_{1}=1,7 \sqrt{\alpha} \\
& \mathrm{c}_{2}=2,12 \frac{\alpha \sqrt{\alpha}}{d^{2}} \\
& \mathrm{c}_{3}=\frac{\operatorname{sh} \bar{\beta}+\sin \bar{\beta}}{\operatorname{ch} \bar{\beta}-\cos \bar{\beta}}
\end{aligned}
$$

Then the expression (14) will take next form:

$$
\psi=\frac{\mathrm{c}_{1} r}{\mathrm{c}_{2} r^{2}+\mathrm{c}_{3}}
$$

Calculating the derivative $\frac{d \psi}{d r}$ and using Fermat's theorem, we obtain

$$
\begin{aligned}
& r_{o p t}=\sqrt{\frac{c_{3}}{c_{2}}} \\
& \psi_{e k c}=\frac{c_{1}}{2 c_{3}} \cdot \sqrt{\frac{c_{3}}{c_{2}}} .
\end{aligned}
$$

It is easy to see that (19) defines an extremum of the maximum type (Fig. 4).

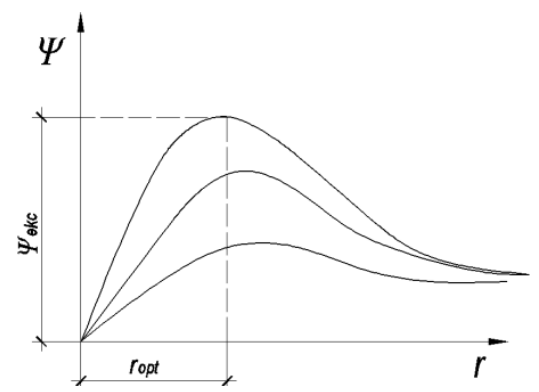

Fig. 4. Dependence $\psi_{(r)}$. 
To evaluate the influence of the distance between the frames on the value of $\psi$, a numerical study was also carried out. Its results are demonstrated by the graphs shown in Fig. 5.

As follows from the graphs, for fixed values of the reduced thickness of the mesh clip $h_{\text {red }}$, the diameter of the frame $d$ and the radius of the concrete core $r$ the value of $\psi=$ const.

Nevertheless, the complete solution of problem (1) $\div(10)$ is of interest.

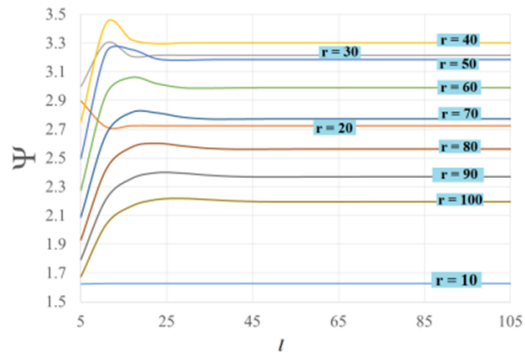

a) $\alpha=0.0125, d=2 \mathrm{~m}$

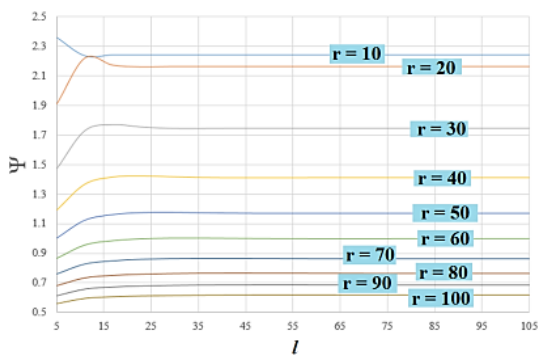

c) $\alpha=0.05, d=2 \mathrm{~m}$

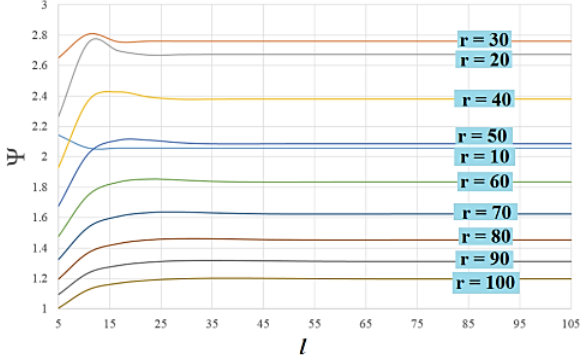

b) $\alpha=0.025, d=2 \mathrm{~m}$

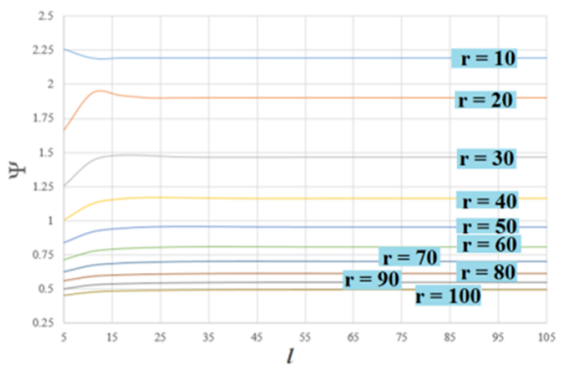

d) $\alpha=0.0625, d=2 \mathrm{~m}$

Fig. 5. Dependence $\psi_{(L)}$.

And, in particular, it is convenient to search for the maximum value and the corresponding values of the components of the vector (8) using the method of integral gradients [4]. In accordance with it, we can write:

$$
\begin{gathered}
\sup \psi=\lim _{p \rightarrow \infty}\left[\int_{\alpha_{\min }}^{\alpha_{\max }} \int_{\gamma_{\min }}^{\gamma_{\max }} \int_{d_{\min }}^{d_{\max }} \psi[\alpha, \gamma, d]^{p} d \alpha d \gamma d d\right]^{1 / p} \\
x_{j}^{*}=\lim _{p \rightarrow \infty} \frac{\int_{\alpha} \int_{\gamma d} \psi[\alpha, \gamma, d]^{p} \chi_{j} \cdot d \alpha d \gamma d d}{\iint_{\alpha} \int_{d} \psi[\alpha, \gamma, d]^{p} d \alpha d \gamma d d}
\end{gathered}
$$

where $x_{j}^{*}$ is a coordinate of the global maximum point, $j=1,2,3, x_{1}^{*}=\alpha ; x_{2}^{*}=\gamma ; x_{3}^{*}=d$.

The numerical solution of (20), (21) for constraints

$$
\begin{aligned}
0.004 & \leq \alpha \leq 0.01 \\
0.25 & \leq \gamma \leq 2.0 \\
0.020 & \leq d \leq 0.036 \\
0.25 \mathrm{~m} & \leq r \leq 1.0 \mathrm{~m}
\end{aligned}
$$

gives:

$$
\psi_{\text {max }}=0.77347 \mathrm{~m}, \alpha_{o p t}=0.06, \gamma_{o p t}=0.25, d_{\text {opt }}=0.0366 \mathrm{~m}
$$


As shown by the analysis of the numerical solution (20), (21), a significant increase in the maximum value of $\psi$ can be obtained, basically, with increasing of the cross-sectional area of the frame $A_{f}$. In particular, for $A_{f}=0.00785 \mathrm{~m}^{2}(\mathrm{~d}=0.1 \mathrm{~m})$ solution of $(20),(21)$ gives the following:

$$
\psi_{\text {max }}=0.150345 \mathrm{~m}, \alpha_{\text {opt }}=0.1, \gamma_{\text {opt }}=0.25, d_{\text {opt }}=0.1 \mathrm{~m} .
$$

Having the expressions (10) and (22), it is possible to estimate the bearing capacity of the concrete core (actually considered support). In this case, the design model of the core should be adopted in accordance with the scheme shown in Figure 2 (sections " 1 - 1" and "2 2 "2").

As criterion for the limiting state, the criterion $[5,6,7,8,9]$ is taken:

$$
e_{i} \leq e_{n},
$$

where $e_{i}$ is a DPED in $i$-s finite element; $e_{n}$ the normalized (limiting) value of the DPED, which is determined by the formula:

$$
e_{n}=0,5 \chi_{\varepsilon}\left[\left(\chi_{\varepsilon}+1\right) e_{n c}+\left(\chi_{\varepsilon}+1\right) e_{n t}\right]+\left(1+W_{\varepsilon}\right) e_{n s h}
$$

$\chi_{\varepsilon}=\frac{2 \varepsilon_{2}-\varepsilon_{1}-\varepsilon_{3}}{\varepsilon_{1}-\varepsilon_{3}}, \varepsilon_{1} \geq \varepsilon_{2} \geq \varepsilon_{3}$ - the main linear deformations;

$\chi_{\varepsilon}$ - Lode-Nadai parameter for strained state

$e_{n c}=\int_{0}^{\varepsilon_{n c}} \sigma_{c} d \varepsilon_{c}, e_{n c}=\int_{0}^{\varepsilon_{n t}} \sigma_{t} d \varepsilon_{t}, e_{n s h}=\int_{0}^{\gamma_{n s h}} \tau d \gamma$.

$\varepsilon_{n c} ; \varepsilon_{n t} ; \gamma_{n s h}$ - normalized (limiting) deformations;

$\sigma_{c}=\sigma_{c}\left(\varepsilon_{c}\right) ; \sigma_{t}=b_{t}\left(\varepsilon_{t}\right) ; \tau=\tau(\gamma)$ - predetermined (known) indicator diagrams of concrete.

Each of the indicator diagrams is represented in the form [10]:

$$
b=a_{1} \varepsilon+a_{3} \varepsilon^{3}+a_{5} \varepsilon^{5}+a_{7} \varepsilon^{7}
$$

where $\{\bar{\alpha}\}=\left\{E_{0}, R, \varepsilon_{R}, \varepsilon_{u}, \beta\right\}$

$E_{0}$ is the initial modulus of deformations;

$R$ is the ultimate strength;

$\varepsilon_{R}$ deformation, corresponding to the ultimate strength;

$\varepsilon_{u}$ is the limiting deformation;

$\beta$ is the stress, corresponding to the limiting deformation.

And the coefficients $\alpha_{j}(j=1,3,5,7)$ are given by Table 1 .

Table 1. Coefficients of a polynomial.

\begin{tabular}{|c|c|}
\hline Coefficient & Value \\
\hline$a_{3} \cdot E_{0}^{-1}$ & $\frac{\varepsilon_{u}^{4}}{2\left(\varepsilon_{u}^{2}-\varepsilon_{R}^{2}\right)^{2}}\left[\frac{1}{\varepsilon_{R}^{2}}\left(\frac{R}{E_{0} \varepsilon_{R}}-1\right)\left(5-7 \frac{\varepsilon_{R}^{2}}{\varepsilon_{u}^{2}}\right)-\frac{1}{\varepsilon_{R}^{2}}\left(\frac{\varepsilon_{R}^{2}}{\varepsilon_{u}^{2}}-1\right)+\frac{2 \varepsilon_{R}^{4}}{\varepsilon_{u}^{6}}\left(\frac{\beta R}{E_{0} \varepsilon_{u}}-1\right)\right]$ \\
\hline$a_{5} \cdot E_{0}^{-1}$ & $\frac{\varepsilon_{u}^{4}}{2\left(\varepsilon_{u}^{2}-\varepsilon_{R}^{2}\right)^{2}}\left[\frac{1}{\varepsilon_{R}^{4}}\left(7 \frac{\varepsilon_{R}^{4}}{\varepsilon_{u}^{4}}-3\right)\left(\frac{R}{E_{0} \varepsilon_{R}}-1\right)-\frac{1}{\varepsilon_{R}^{4}}\left(1-\frac{\varepsilon_{R}^{4}}{\varepsilon_{u}^{4}}\right)-\frac{4 \varepsilon_{R}^{2}}{\varepsilon_{u}^{6}}\left(\frac{\beta R}{E_{0} \varepsilon_{u}}-1\right)\right]$ \\
\hline$a_{7} \cdot E_{0}^{-1}$ & $\frac{\varepsilon_{u}^{4}}{2\left(\varepsilon_{u}^{2}-\varepsilon_{R}^{2}\right)^{2}}\left[\frac{1}{\varepsilon_{R}^{4} \varepsilon_{u}^{2}}\left(\frac{R}{E_{0} \varepsilon_{R}}-1\right)\left(3-5 \frac{\varepsilon_{R}^{2}}{\varepsilon_{u}^{2}}\right)-\frac{1}{\varepsilon_{R}^{4} \varepsilon_{u}^{2}}\left(\frac{\varepsilon_{R}^{2}}{\varepsilon_{u}^{2}}-1\right)+\frac{2}{\varepsilon_{u}^{6}}\left(\frac{\beta R}{E_{0} \varepsilon_{u}}-1\right)\right]$ \\
\hline
\end{tabular}

While calculating (comparing) (23), it is assumed that the solution of the problem is constructed using finite element method [4]. Here as the environment for the realization of 
calculation the "Lira" Software Package was used, which allows determining the value of $e_{i}$.

\section{Conclusion}

Analysis of these calculations shows that the increase in the bearing capacity of a short pillar due to the use of a rational mesh clip without frames is $20 \%$, with frames $-35 \%$.

As a consequence, it can be noted that the theoretical results which were obtained are fully justify the effectiveness of the proposed solution.

\section{References}

1. V.S. Shmukler, E.A. Petrova, E.V. Sinkovskaya. Scientific Bulletin of Building, Kharkiv, HNUBA, HOTW ABU, 74, 148-156 (2013)

2. V.G Rekach. Guidance for solving problems of the theory of elasticity (Moscow: Higher School, 1966)

3. S.P. Timoshenko. Resistance of materials, v.2. More complex theoretical questions and problems (Moscow. "Science" Publishing House, 1965)

4. A.S. Gorodetsky, V.S. Shmukler, A.V. Bondarev. Information technology of calculation and design of building structures (Kharkov, NTU "KhPI", 2003)

5. G.V. Vasilkov. Evolutionary problems of structural mechanics. Synergetic paradigm (Rostov-on-Don, Infoservis, 2003)

6. G.V. Vasilkov. Evolutionary theory of the life cycle of mechanical systems. Theory of Structures (Moscow, LKI, 2008)

7. V.S. Shmukler, Y.A. Klimov, N.P. Burak. Frame systems of a light type (Kharkov, Golden Pages, 2008)

8. V.S. Shmukler. Proceedings of the International conference, Scotland, 183-190 (2008)

9. V.S. Shmukler. Third International Structural Engineering and construction Conference, Shunan, Japan, 539-545 (2005)

10. V.S. Shmukler. Collected Scientific Works of Ukrainian State University of Railway, UkrDUZT, 167, 54-69 (2016) 